Check for updates

NHS Confederation

Cite this as: $B M J 2022 ; 376: 018$ http://dx.doi.org/10.1136/bmi.o18 Published: 06 January 2022

\section{Omicron: Urgent action needed on NHS staffing crisis}

\section{We are now in a position where a small amount of extra pressure could be the final straw, writes Layla McCay}

Layla McCay director of policy

NHS leaders across the UK are currently looking nervously at London. The omicron wave that has (hopefully) plateaued in England's capital city is building throughout the rest of the country, and everyone wants to know what to expect. There is no easy answer to that. Those who were looking at South Africa in the early days of omicron's spread have recognised how hard it can be to compare such different populations. But closer to home, particularly in London, insight can be found from the massive number of people who have caught the omicron variant of covid-19 over the past few weeks.

So, what do we now know?

Thankfully the proportion of infected people who have thus far needed hospital care has been lower than with previous variants, and those admitted to hospital have tended to need less intensive care. But a small proportion of a massive number is still a lot of people. Cases rose by 50\% between 28 December 2021 and 3 January 2022 compared with the week before. One 189985 people tested positive for covid over that week, with the Office of National Statistics estimating that 1 in 15 people in England had covid-19 in the week to 31 December 2021, and 1 in 10 in London. ${ }^{1}$ With a further rise in diagnoses reported this week, numbers continue to soar above anything we have seen before.

For all the speculation, there is no way to know for sure exactly how omicron will affect the population or the NHS. The variant is still relatively new, and we have limited data about its behaviour in different population groups, particularly in older people who were infected in smaller numbers before Christmas, but have been more likely to catch it over the festive period. Early reports suggest that this cohort may be more likely to need hospital care. Despite our incredible vaccination programme, our immunity as a nation is complex and patchy, with vaccine uptake patterns mirroring and contributing to health inequalities.

What has distinguished omicron from other variants of SARS-CoV-2 so far is that the challenges have so far not been as focused on intensive care as previous waves. Certainly, some people with the omicron variant are in intensive care. But, thanks to a combination of vaccination and previous infection in the population, behaviours taken to reduce transmission, factors relating to the virus itself, and new treatment protocols, hopefully only a smaller proportion of the population will be severely ill from omicron than with delta.

That said, hospital admissions for covid-19 are rising, both in terms of people needing care due to the virus, and people needing healthcare for other reasons, but happening to test positive for covid-19, necessitating the same onerous infection control protocols that take up considerable extra time and space, reduce capacity, and put extra pressure on all parts of the system. More than 15000 people are currently in hospital with covid-19 and that number is still rising. What is different in this wave compared to those we have previously experienced is the concurrent high demand for other urgent and emergency care, plus the work being done to address the elective backlog. Further, capacity challenges in domiciliary, community, and social care mean discharge barriers, reducing capacity for new admissions. That this omicron wave coincides with what has historically been the busiest time of the year for the NHS only compounds the challenge.

As demand for covid-19 care (as well as for other urgent, emergency, and planned care) continues to rise across the system, NHS leaders find themselves with another critical supply and demand imbalance. A huge reason that the omicron wave is proving so disruptive to the NHS is that NHS staff are catching it along with everyone else. Across the country, more staff are now off due to covid-19, whether sick or isolating, than at any other point of the pandemic. This clearly varies by location and service, but some hospital leaders have described having 1 in 10 of their staff off sick right now.

Some services are disrupted; some cannot currently be delivered. This is all putting further pressure on those staff who are not yet absent and who are in many cases working beyond the call of duty-following two years of intense pressure-to keep services running. The focus must be on finding ways to maintain adequate staffing across the system, from ensuring an uninterrupted supply of tests, to seeking national review of appropriate isolation periods, to mobilizing relevant cohorts to support current staff, and ensuring regulators recognise interim changes to ways of working required to meet these exceptional circumstances. Innovative out-of-hospital care approaches will also be helpful.

We are now in a position where a small amount of extra pressure could be the straw that breaks the camel's back. Hopefully this will be a brief wave and smoother seas are on the horizon. But if omicron has a more severe impact on the older population who are increasingly being affected, if it hits areas where there are lower vaccination levels particularly hard, if more staff become absent, and if flu season kicks off, the NHS will be in an even more challenging situation. 


\section{OPINION}

Provenance and peer review: commissioned, not peer reviewed

1 Office for National Statistics. Coronavirus (COVID-19) Infection Survey headline results, UK: 5 January 2022. https://www.ons.gov.uk/peoplepopulationandcommunity/healthandsocialcare/conditionsanddiseases/bulletins/coronaviruscovid19infectionsurveypilot/5january2022 